\title{
Agency in the digital age: Using symbiotic agency to explain human-technology interaction
}

\author{
Gina Neff \\ Oxford Internet Institute \& Department of Sociology, University of Oxford \\ Peter Nagy \\ Arizona State University \\ To appear in A Networked Self: Human Augmentics, Artificial Intelligence, Sentience, \\ Zizi Papacharissi, ed. Routledge, 2018.
}

\section{Introduction}

Recent advancements in technology challenge our fundamental notions of human power and agency. Tools and techniques including machine learning, artificial intelligence, and chatbots may be capable of exercising complex "agentic" behaviors (Dhar, 2016). Advanced technologies are capable of communicating with human beings in an increasingly sophisticated manner. Ranging from artificial chat partners through the commercial algorithms of social media to cutting-edge robots, these encounters with interactive machines often result in a complex and intimate relationship between users and technologies (Finn, 2017). For instance, people now may have their own virtual assistants such as Apple's Siri and Amazon's Alexa. Other commercial technological agents "help" people find new movies on Netflix or friends on Facebook. Robotic companions, like Huggable developed by Cynthia Lynn Breazeal at MIT, can read human emotions and react to them accordingly. Chatbots have long evoked reactions from people that can be used therapeutically for psychological counselling and now these tools are being rolled 
out as apps to help people cope with anxiety and depression (Lien, 2017; Neff and Nagy, 2016). Such developments present a quandary for scholars of communication. Does the agency of the people and, increasingly, of things that we chose to communicate with matter? This question prompts us to urge communication scholars to develop a better definition of agency and more clarity on how agency is enacted in practice within complex, technologically-mediated interactions.

Agency has been an inherently fuzzy concept, referring to different concepts depending on the particular discipline where it is being used. Campbell (2005) notes that "agency is a polysemic and ambiguous, a term that can refer to invention, strategies, authorship, institutional power, identity, subjectivity, practices, and subject positions, among others" (p. 1). Agency has long connoted a distinctive human ability and something that by definition only humans possess. Theories connecting agency to intentionality (Jansen, 2016) and to the capacity to achieve one's goals (Caston, 2011) amplify this sense of meaning of agency. The idea of agency as inherently social and cooperative is constrained by the symbolic and material elements of context and culture (Campbell, 2005).

Recent social theories have started to analyze the different ways nonhumans, like technologies, may acquire agentic capacities and quasi-agentic abilities. For instance, when discussing the pervasive social implications of these emerging technological artifacts, communication and media studies scholars often focus on how and for what people use these tools. As such, they emphasize the role of social context and/or affordances in technology usage (e.g. Balsamo, 2011; Cooren, 2010; Siles and Boczkowksi, 2012). Yet, communication and media scholars tend to neglect the different ways users imagine and communicate with these interactive social agents (Guzman, 2016; Leonardi \& Barley, 2008). Science and technology 
studies (STS) scholars have already mapped how human and technological agents interact with each other. Bruno Latour (1999) from the Actor-Network Theory (ANT) movement, and François Cooren (2010) from the Montreal School of organizational communication have theorized the tensions between human and nonhuman actors. Similarly, communication and media scholars have focused on the different ways users derive meaning from encounters with technologies capable of exercising a wide range of agentic behaviors (e.g. Grossberg, 1997; Foss et al., 2007). What these conceptualizations still miss is that they have not addressed the ontological differences, and similarities, between human and nonhuman agency (Jansen, 2016).

As a corrective, we reframe and redefine agency in terms of how and when people interact with complex technological systems. For this we turn to Albert Bandura's socialcognitive psychological theory of human agency and to the concept of symbiosis in biology. Bandura's agency theory helps us understand the role of users in shaping and determining technical systems. Being an agent means that people can exert intentional influence over their mental processes and physical actions to achieve their desired outcomes (Bandura, 2006). People, however, rarely act on their own. They tend to delegate tasks to sophisticated technologies (e.g. smartphones, computers, etc.), often viewing them as personal assistants and companions rather than simple tools. Rod and Kera (2010) have specifically used the term symbiosis for our increasing interdependent relationships with technologies. The theory that we extend with this chapter imagines human-technology interaction as a symbiotic interplay of human and nonhuman agents, further blurring the line between what users want to do and what technologies are capable of doing. In the present chapter, we will introduce our symbiotic agency theory and show how it can address the problems around agency theories developed by communication and media studies as well as in science and technology studies. 


\section{Conceptualizing agency in human-technology interactions: an unfinished project}

Agency is generally viewed as a capacity to act, produce and anticipate a desired outcome within a particular context (Giddens, 1979; Wang, 2008; Leonardi, 2011). This contextual element to scholarly definitions mean agency is "communal, social, cooperative, and participatory and, simultaneously constituted and constrained by the material and symbolic elements of context and culture" (Campbell, 2005, p. 3). Communication theories tend to focus on exploring how users construe meaning through reflective and symbolic manipulation of technologies (Siles \& Boczkowski, 2012). As such, communication and media scholars have made a real progress in moving beyond the former simplistic views of human-technology interaction in which humans are always in control and technologies do not exert influence over their users (Orlikowski, 2005; Lievrouw, 2013; Guzman, 2016). However, communication and media studies theories often stop short of conceptualizing interdependent, entwining human and nonhuman agencies, and instead focus on the agentic dimensions of socio-technical systems (e.g., Leonardi \& Barley, 2008).

For STS scholars agency is typically seen as people's general ability to shape the material form and meaning of artifacts through practices and interactions (Pickering, 1995; Feenberg, 1999; Gillespie, 2006). The STS discourse discusses agency in conjunction with materiality or the content of media technologies, framing agency as a dynamic notion centered around how users create and interpret as well as shape and attach content to media technologies. As Latour (1999) phrased this, "we are sociotechnical animals and each human interaction is sociotechnical" (p. 214). By this he meant people delegate tasks to technologies, enrolling them into larger scripts for action. Traffic speed bumps take the place of police officers standing at intersections, brought into action by a series of connected links to urge drivers to slow down. 
From Latour's perspective, agency can always be best understood as a complex interplay of nonhumans and humans in these larger systems. Humans make nonhumans act on their behalf, but nonhumans also do the same with humans (Hannemyr, 2003).

The larger project of STS and ANT deepened our understanding of the social dimensions and social "lives" of technologies. Both communication and STS scholars have shown how agency is embedded in a broader socio-cultural landscape. It also led, unfortunately, to what we might argue are simplified explanations of the role of technology as a social force, and a confusing conceptualization of agency. Bringing in language to address the multifaceted nature of human agency and its connections to technological agents would help correct this.

Our pragmatic working definition of agency is what users, actors and tools, $d o$ when interacting with complex technological systems. This means that agency is closely linked to intentionality (Jansen, 2016) and to the capacity to achieve one's goals (Caston, 2011). Agency can mobilize people to reflect on their experience, adapt to their surroundings, and affect the sociocultural context that in turn sustains them (Jenkins, 2008). From this starting point, we extend the notion of agency by combining Albert Bandura's agency theory and the concept of symbiosis. This will help scholars develop a more nuanced and detailed understanding of how people think, feel, and act when interacting with the technical.

\section{Defining human agency from a social-cognitive perspective}

Albert Bandura, one of the most influential social-cognitive thinkers, considers human agency a central force in human functioning (Bandura, 1989). Being an agent means that people can exert intentional influence over their mental processes and physical actions to achieve their desired outcomes (Bandura, 2000). Agency enables individuals to exercise greater control over their mental functioning, and to monitor how their behaviors affect the world around them (Bandura, 
2001). The social cognitive perspective holds that human agency is built around four core properties (Bandura, 2006). One is intentionality referring to action plans and potential strategies people form to achieve their goals and aspirations. The second property is forethought, plans humans fabricate for the future, helping them set goals and anticipate likely outcomes; thus forethought plays important role in guiding and motivating people's efforts. In addition, agents are not only planners, but also self-regulators who are capable of managing their emotions and cognitions. Finally, the last component, self-reflectiveness enables human beings to examine their own functioning through reflecting on their feelings, thoughts and behaviors.

Since individuals are rarely act on their own, social psychological theories also investigate the cognitive, motivational or affective processes of agency not only on a personal but also on a social level. In addition to the previously discussed personal agency, people may exercise proxy and collective agency as well (Bandura, 2000). Whereas collective agency represents people's shared beliefs in accomplishing goals through cooperative efforts, proxy agency is enacted through different processes (Bandura, 2006). Proxy agency refers to a sociallymediated mode that involves other individuals or tools that can act on their behalf, and can help someone to achieve goals and desirable outcomes. In this sense, proxy agency heavily involves the mediative efforts of others (Bandura, 2001). Finally, human beings mainly turn to proxy control to exert their influence when they lack of developed means to do so or when they believe others can perform better (Bandura, 2002).

Proxy agency becomes a particularly powerful lens for looking at the relationships between users and tools. Consider the example of mobile phones. They require regular maintenance that necessitates human interventions such as. charging the batteries, switching on/off, and paying the bills. But mobile phones also assist their users by reminding them about 
important upcoming events, providing information on issues, and ultimately helping them better organize their social lives. People can "outsource" their concerns about themselves to their devices, allowing their smartphones and other trackers to assist them in their efforts to get more sleep, more exercise, or fewer calories (Schull, 2016). The concept of proxy agency helps to frame these desires and interactions as a form of power of remaking and reshaping the world that people and their tools attribute to one another.

By borrowing the idea of symbiosis, we illustrate the ways that fundamental biology concepts may help us conceptualize this entanglement of human and technological agency when we talk about complex socio-technological systems.

\section{The concept of symbiotic agency}

The term 'symbiosis' derives from the Greek word 'living together'. It was first coined by the botanist, Anton de Bary, in the mid-nineteenth century who defined symbiosis as "the living together of different species" (Sparrow, 1978). As Sapp (1994) put it "we evolved from, and are comprised of, a merger of two or more different kinds of organisms living together. Symbiosis is at the root of our being" (p. xiii). As such, symbiosis is often viewed as a virtually ubiquitous phenomena serving as the basis of normal development (Pradeu, 2011). Forming symbiosis may help organisms augment their robustness and functionalities (Kitano and Oda, 2006) and acquire new capacities (Douglas, 1994).

Psychologically informed research also viewed symbiosis as an essential concept and considered it a central force in orchestrating our relationship with the social world (Chodorow, 1989; Tissaw, 2000). Rather than being passive recipients, individuals are are actively involved in their social development via taking part in symbiotic relationships. The idea of symbiosis can be applied to human-technology relationship by illustrating the fact that people use technologies 
for augmenting their capacities or acquiring new ones. The previously discussed core properties of agency coined by Bandura (2006) can help us understand how symbiotic agency is formed between users and technologies.

In terms of intentionality, when individuals use their cell phones as alarm clocks to wake them up in the morning, they rely on the perceived nonhuman agentic properties and affordances of mobiles. The user's intention to wake up in time is delegated to the cellphone, in other words, they practice proxy agency. Forethought, on the other hand, gives us valuable insights into how users' future-oriented and anticipatory behaviors may be supported and extended by technological artifacts. For example, some individuals regularly use health-trackers or other related applications in hope to take full control over their biological functioning. They do it because they would like to preserve their health, or prevent diseases. In other words, they use devices or applications as temporal extensions of their agencies. Again, similarly to intention, users delegate the forethought dimension of their agency in order to regulate their life, in this case their health, more effectively. They also use technological artifacts in hope to create coherence and find meaning in their lives (Neff \& Nafus, 2016), but perhaps more importantly, because health-trackers and related applications are interactive entities. The third agentic component, self-regulation (or as Bandura calls it self-reactiveness) points out to the motivational and affective aspects of technology usage. For instance, several devices and programs are constructed to promote healthy behavior for users (e.g. quit smoking or healthy eating applications), for these artifacts send feedback on one's behavior and 'performance' on a regular basis mobilizing the motivational properties of agency. Human-technology interaction is characterized both by users' perceptions and emotions as well as the emotions evoked by the design and aesthetic features of devices or digital products (Mick \& Fournier, 1998; Thüring \& 
Mahlke, 2007). Finally, with the emergence of sophisticated artificial agents, users gained ample opportunities to reflect on nearly every aspects of their lives (e.g. working, learning or socializing). When applied to human-technology interaction, self-reflectiveness practices have been changed, shaped and modified by technological artifacts due to the mediative characteristics of these tools. That is, users may explore technological artifacts socially, culturally, and cognitively so they can experience different modes of agentic functioning and self-reflective practices.

Tracing the forms of agency that people can have within technological systems, we propose the term symbiotic agency referring to a specific form of proxy agency that users and tools can enact within human-technology interaction. We argue that symbiotic agency can be considered as a form of soft determinism encompassing both how technology mediates our experiences, perceptions and behavior, and how human agency affects the uses of technological artifacts. More specifically, we propose that when individuals interact with technologies, they exercise proxy agency through a technologically mediated mode referring to the entanglement of human and nonhuman agencies. For symbiosis, similarly to entanglement, implies an obligated relationship - symbionts are completely dependent on each other for survival, it can be considered a proxy agentic relationship that may provide different benefits for the recipients.

\section{The different forms of symbiotic agency}

Previous biological research also showed that symbiosis is a complex phenomena; organisms may benefit from, be harmed by, or not be affected by symbiotic relationships (Paracer and Ahmadjian, 2000). An association in which one symbiont benefits and the other is neither harmed nor benefited is a commensalistic symbiosis. An association in which both symbionts 
benefits is a mutualistic symbiosis. A relationship in which a symbiont receives nutrients at the expense of a host organism is a parasitic symbiosis. The conceptual boundaries, however, are not clear, and there are frequent transitions between the three forms of symbiosis (Tissaw, 2000). The different forms of symbiosis can be also used as lens for understanding how users' expectations, beliefs and values may influence their proxy agentic relationship with technologies.

People have a general tendency to anthropomorphize technologies that are often designed to elicit a wide range of projections (Darling, 2014). The "Uncanny Valley Effect" (Reichardt, 1978) documents the discomfort that people feel when robotic agents are seen as having too human-like appearance, and this effect is even more intense when a particular robot has some form of disfigurement or abnormal feature (Seyama \& Nagayama, 2007). When ascribing agency to complex technologies, such as chatbots, robots or other AI applications, people project their emotions to these entities and try to find explanations for their "behaviors" (Darling, 2014). A recent study by Neff and Nagy (2016) already showed that when users interact with chat programs or chatbots they tend to attribute "personality" to the technical, and project a wide range of emotions to them, that in turn change how the artifact communicates with them. Similarly, within media psychology, "computers as social actors" theory (Nass \& Moon, 2000) holds that users unconsciously apply social rules to technologies and interact with them as they were living organisms. The ways individuals approach these technologies can be traced further back to their general tendency to treat simple things as autonomous and intelligent entities (Shermer, 2008). For instance, in Fritz Heider's and Marianne Simmel's (1944) classic experiment, participants were asked to watch a short animation about triangles and circles moving in weird patterns. Surprisingly, when instructing the subjects to describe what they saw, Heider and Simmel found that people fabricated complete and detailed stories about the 
geometric figures which they treated as agents and ascribed various emotional states and goals to them. These examples show why people may view their relationship with technologies as a form of commensalistic symbiosis and treat devices as simple tools enabling them to achieve their goals. Similarly, users may feel that they form mutualistic relationship with technologies, such as chatbots or robots that simultaneously help them as well as learn from them. Finally, users may also feel that they are increasingly getting "addicted to" or "controlled by" technologies due to the perceived parasitic attributes of these tools.

Symbiotic agency and its different forms show us that human-technology relationship is no longer related solely to human action but also to technological action. Our concept represents the entanglement of human and technological agencies. We have argued here that symbiotic agency is a more appropriate expression to capture the dynamic nature of human and technological agency in human-machine communication, in which users simultaneously influence and being influenced by technological artifacts.

\section{The implications of symbiotic agency for human-technology interaction}

By building on our working definition of agency, we proposed a new theoretical framework for studying human-technology interaction. Our theory presents human-technology interaction as a symbiotic interplay of human and nonhuman agents, further blurring the line between what users want to do and what technologies are capable of doing. By combining psychological as well as communication and media studies concepts, we proposed that scholars can build a better theory for studying complex communication technologies. Technological artifacts have an impact on their users because of their interactive features and affordances (Nagy \& Neff, 2015), and individuals' agentic properties shape the uses of artifacts for accomplishing goals and creating 
meaning. Reimagining agency this way brings meaning making into more arenas of social and technological life.

Symbiotic agency also helps scholar better capture the intimate linkages among human and technical agents. Ranging from artificial chat partners through the commercial algorithms of social media to cutting-edge robots, today's world is now filled with advanced technologies that are capable of communicating with human beings in a sophisticated manner (Guzman, 2016). When studying socio-technical systems, symbiotic agency allows scholars move beyond simplistic terms of the debate about social constructivism vs. technological determinism, and better conceptualize the encounters between human and nonhuman. With the notion of symbiotic agency, scholars are able to address and explore the dynamic dimensions of human-technology relationship. By redefining human-technology interaction as a unique form of proxy agency, media and communication theorists can investigate both the human agentic properties as well as the technological attributes of what it means to be a networked self.

\section{Acknowledgements}

This material is based upon work supported by the National Science Foundation under Grant No. 1516684.

\section{References}

Arbib, M. A. (1974). Man-Machine Symbiosis and the Evolution of Human Freedom. The American Scholar, 43(1), 38-54.

Balsamo, A. (2011). Gendering the Technological Imagination. Durham, NC: Duke University Press.

Bandura, A. (1989). Human Agency in Social Cognitive Theory. American Psychologist, 44(9), 1175-1184. 
Bandura, A. (2000). Exercise of Human Agency Through Collective Efficacy. Current Directions in Psychological Science, 9(3), 75-78.

Bandura, A. (2001). Social Cognitive Theory: An Agentic Perspective. Annual Review of Psychology, 52, 1-26.

Bandura, A. (2002). Growing Primacy of Human Agency in Adaptation and Change in the Electronic Era. European Psychologist, 7(1), 2-16.

Bandura, A. (2006). Toward a Psychology of Human Agency. Perspectives on Psychological Science, 1(2), 164-180.

Buchner T (2016) 'Machines don't have instincts': Articulating the computational in journalism. New Media \& Society, 19(6), 918-933.

Campbell, K. K. (2005). Agency: Promiscuous and Protean. Communication and Critical/Cultural Studies, 2(1), 1-19.

Caston, J. (2011). Agency as a psychoanalytic idea. Journal of the American Psychoanalytic Association, 59(5), 907-938.

Chodorow, N. J. (1989). Feminism and Psychoanalytic Theory. London, UK: Yale University Press.

Cooren, F. (2010). Action and Agency in Dialogue: Passion, incarnation and ventriloquism. Philadelphia, PA: John Benjamins Publishing Company.

Dar, V. (2016). The Future of Artificial Intelligence. Big Data, 4(1), 5-9.

Darling, K. (2014). Extending Legal Protection to Social Robots: The effects of Anthropomorphism, Empathy, and Violent Behavior Towards Robotic Objects. In R. Calo, M. Froomkin, \& I. Kerr (Eds.), Robot Law (pp. 212-232). Camberley Surrey: Edward Elgar Publishing.

Douglas, A. E. (1994). Symbiotic Interactions. Oxford, UK: Oxford University Press.

Feenberg, A. (1999). Questioning Technology. New York, NY: Routledge. 
Finn, E. (2017). What Algorithms Want: Imagination in the Age of Computing. Cambridge, MA: MIT Press.

Foss, S. K., Waters, W. J. C., \& Armada, B. J. (2007). Toward a Theory of Agentic

Orientation: Rhetoric and Agency in Run Lola Run. Communication Theory, 17(3), 205-230.

Hannemyr, G. (2003). The Internet as hyperbole: A critical examination of adoption rates. The Information Society, 19(2), 111-121.

Heider, F., \& Simmel, M. (1944). An Experimental Study of Apparent Behavior. The American Journal of Psychology, 57, 244-259.

Giddens, A. (1979). Central problems in social theory: Action, structure, and contradiction in social analysis. London, UK: Macmillan.

Gillespie, T. (2006). Designed to "effectively frustrate": Copyright, technology and agency of users. New Media \& Society, 8(4), 651-669.

Grossberg, L. (1997). Bringing it all back home: Essays on cultural studies. Durham, NC: Duke University Press.

Gunkel, D. (2000). We Are Borg: Cyborgs and the Subject of Communication.

Communication Theory, 10(3); 332-357.

Guzman, A. (2016). The Messages of Mute Machines: Human-Machine Communication with Industrial Technologies. Communication $+1,5$, Article 4 .

http://scholarworks.umass.edu/cgi/viewcontent.cgi?article=1054\&context=cpo.

Jansen, T. (2016). Who is Talking? Some Remarks on Nonhuman Agency in Communication. Communication Theory, 26(3), 255-272.

Jenkins, A. H. (2008). Psychological Agency: A Necessarily Human Concept. In Frie, R. (Ed.). Psychological Agency: Theory, Practice, and Culture (pp. 177- 200). Cambridge, MA: MIT Press. 
Kitano, H., and Oda, K. (2006). Self-Extending Symbiosis: A Mechanism for Increasing Robustness Through Evolution. Biological Theory, 1(1), 61-66.

Latour, B. (1999). On recalling ANT. The Sociological Review, 47(1), 15-25.

Leonardi, P. M. (2011). When flexible routines meet flexible technologies: Affordance, constraint, and the imbrication of human and material agencies. MIS Quarterly, 35(1), 147167.

Leonardi, P. M., \& Barley, S. R. (2008). Materiality and change: Challenges to building better theory about technology and organizing. Information and Organization, 18(3), 159-176.

Licklider, J. C. R. (1950). Man-Computer Symbiosis. Ire Transactions on Human-Factors in

Electronics, Retrieved from http://worrydream.com/refs/Licklider\%20-\%20Man-

Computer\%20Symbiosis.pdf.

Lien, T. (2017). Depressed but can't see a therapist? This chatbot could help. Retrieved from http://www.latimes.com/business/technology/la-fi-tn-woebot-20170823-

htmlstory.html?utm_campaign=KHN\%3A\%20First\%20Edition\&utm_source=hs_email\&utm _medium=email\&utm_content=55559892\&_hsenc=p2ANqtz-

8OaMwjlJ3xD6NliosxXQXAwYWuYGCObgZbgysXOFjQlewbzhp8yJ3ueYtP2X1Qu1NZD

EFj7LyuTD6A9FBLTI_YcBCm0w\&_hsmi=55559892

Lievrouw, L. (2013). Materiality and media in communication and technology studies: An unfinished project. In T. Gillespie, P. Boczkowski, \& K. Foot (Eds.), Media Technologies: Essays on Communication, Materiality, and Society (pp. 21-52). Cambridge, MA: MIT Press.

Marcus, G. E., \& Saka, E. (2006). Assemblage. Theory, Culture \& Society, 23(2-3), 101-109.

Meyer, E. T. (2015). The expert and the machine: Competition or convergence? Convergence:

The International Journal of Research into New Media Technologies, 21(3), 306-313. 
Mick, D. G., \& Fournier, S. (1998). Paradoxes of technology: Consumer cognizance, emotions, and coping strategies. Journal of Consumer Research, 25(2), 123-143.

Nagy, P. \& Neff, G. (2015). Imagined affordances: Reconstructing a keyword for communication theory. Social Media + Society, Retrieved from http://sms.sagepub.com/content/1/2/2056305115603385.full

Neff, G., \& Nafus, D. (2016). Self-tracking. Cambridge, MA: MIT Press.

Neff, G., \& Nagy, P. (2016). Talking to Bots: Symbiotic Agency and the Case of Tay. International Journal of Communication, 10, 4915-4931.

Nass, C., \& Moon, Y. 2000. Machines and mindlessness: Social responses to computers. Journal of Social Issues, 56(1), 81-103.

Orlikowski, W. J. (2005). Material Works: Exploring the Situated Entanglement of Technological Performativity and Human Agency. Scandinavian Journal of Information Systems, 17(1), 183-186.

Paracer, S., \& Ahmadjian, V. (2000). Symbiosis: An Introduction to Biological Associations. Oxford, UK: Oxford University Press.

Pickering, A. (1995). The Mangle of Practice: Time, Agency, and Science. Chicago, IL: The University of Chicago Press.

Pradeu, T. (2011). A Mixed Self: The Role of Symbiosis in Development. Biological Theory, $6,80-88$.

Reichardt, J. (1978). Robots: fact, fiction and prediction. London, UK: Thames \& Hudson.

Rod, J., \& Kera D. (2010). From agency and subjectivity to animism: phenomenological and Science Technology Studies (STS) approach to design of large techno-social systems. Digital Creativity, 21(1), 70-76.

Sapp, J. (1994). Evolution by Association: A History of Symbiosis. Oxford, UK: Oxford University Press.

Schull, N. (2016). Data for life: Wearable technology and the design of self-care. BioSocieties. 11. . 10.1057/biosoc.2015.47.

Seyama, J., \& Nagayama, R. S. (2007). The Uncanny Valley: Effect of Realism on the Impression of Artificial Human Faces. Presence, 16(4): 337-351. 
Shermer, M. (2008). Patternicity: Finding Meaningful Patterns in Meaningless Noise: Why the brain believes something is real when it is not. Retrieved from http://www.scientificamerican.com/article/patternicity-finding-meaningful-patterns/. Siles, I., \& Boczkowski, P. (2012). At the Intersection of Content and Materiality: A TextoMaterial Perspective on the Use of Media Technologies. Communication Theory, 22(3), 227249.

Sparrow, F. K. (1978). Professor Anton de Bary. Mycologia, 70, 222-252.

Thüring, M., \& Mahlke, S. (2007). Usability, aesthetics and emotions in human-technology interaction. International Journal of Psychology, 42(4), 253-264.

Tissaw, M. A. (2000). Psychological Symbiosis: Personalistic and Constructionist Considerations. Theory \& Psychology, 10(6): 847-876.

Wang, Y. (2008). Agency: The internal split of structure. Sociological Forum, 23(3), 481-502. Wise, M. J. (1998). Intelligent Agency. Cultural Studies, 12(3), 410-428. 